\title{
sciendo
}

Int. J. of Applied Mechanics and Engineering, 2021, vol.26, No.2, pp.1-10

DOI: 10.2478/ijame-2021-0016

\section{DYNAMIC VIBRATION EXTINGUISHED ON A VISCOUSLY ELASTIC BASE}

\author{
M. ISHMAMATOV* \\ Navoi State Mining Institute, Higher Mathematics Department, Galaba Shokh Street, Navoi, UZBEKISTAN \\ E-mail: matlab1962@mail.ru \\ N. KULMURATOV \\ Navoi State Mining Institute, Mechanical Engineering Department, Galaba Shokh Street, Navoi \\ UZBEKISTAN \\ S. KHALILOV and N. AKHMEDOV \\ Navoi State Mining Institute, Higher Mathematics Department, Galaba Shokh Street, Navoi, UZBEKISTAN
}

\begin{abstract}
The aim of the work is to develop algorithms and a set of programs for studying the dynamic characteristics of viscoelastic thin plates on a deformable base on which it is installed with several dynamic dampers. The theory of thin plates is used to obtain the equation of motion for the plate. The relationship between the efforts and the stirred plate obeys in the hereditary Boltzmann Voltaire integral. With this, a system of integro-differential equations is obtained which is solved by the method of complex amplitudes. As a result, a transcendental algebraic equation was obtained to determine the resonance frequencies, which is solved numerically by the Muller method. To determine the displacement of the point of the plate with periodic oscillations of the base of the plate, a linear inhomogeneous algebraic equation was obtained, which is solved by the Gauss method. The amplitude - frequency response of the midpoint of the plate is constructed with and without regard to the viscosity of the deformed element. The dependence of the stiffness of a deformed element on the frequency of external action is obtained to ensure optimal damping of vibrational vibrations of the plate.
\end{abstract}

Key words: dynamic dampers, oscillations, viscoelastic plate, integro-differential equation, amplitude-frequency dependence, Boltzmann-Voltaire integral.

\section{Introduction}

The transformation in machines and mechanisms of some types of energy into others, the transformation of forms of movement, the implementation of work processes are inevitably associated with the emergence of variable forces that generate vibration $[1,2,3]$. It negatively affects the strength and reliability of the operation of machines, load-bearing structures and has a harmful effect on the physiological state of people $[4,5]$. During the operation of electrical machines, vibrations are often observed during their operation [6]. Oscillations can be caused by disturbing forces of mechanical, electrical and aerodynamic origin. By balancing the rotor, improving the suspension and design of an electric machine, it is not always possible to reduce the level of vibrations to permissible norms, and therefore additional means have to be found to damp unwanted vibrations $[7,8]$. In order to limit vibration in various fields of technology, there are requirements and standards for its regulation. In most cases, the norms are established taking into account all the most important conditions and, since they cannot equally satisfy all the requirements, they are the result of a compromise solution $[9,10,11,12]$. Dynamic vibration damping consists in attaching a system to the protected object, the reactions of which reduce the vibration range of the object at the points of connection of this system.

If the frequency of the disturbing force changes little, then one of the most promising, still ways to reduce

\footnotetext{
${ }^{*}$ To whom correspondence should be addressed
} 
the level of vibrations is the use of dynamic absorbers [13, 14]. A dynamic damper is schematically a mass suspended by a spring and having the ability to move in one or more directions. It is known that the use of a damper tuned to the frequency of the disturbing force makes it possible to stop the movement of a body with one degree of freedom at this frequency and to reduce the level of vibrations at frequencies close to it $[15,16,17]$. Currently, the simplest, most affordable and widespread vibration isolation means are elastic elements and dynamic vibration dampers. At sufficiently high frequencies, the elastic elements provide reflection of most of the vibrational energy back to the source, and the better, the lower their rigidity. In the low frequency range, the requirements for the stiffness value are, as a rule, completely different. They are determined by the static load, its changes when tilting, as well as inertial forces during acceleration and deceleration on moving objects, impacts, centering of the mechanism and other operating conditions [18, 19, 20].

In this paper, we consider the problem of reducing the vibration level of a plate on a viscoelastic foundation using dynamic vibration dampers.

\section{Formulation of the problem}

Consider the vibrations of a rectangular plate with length $l$ and width $b$, contacting its entire surface with an elastic base and pivotally supported along the longitudinal edges (Fig.1). We will take the middle plane of the plate as the coordinate plane $x y$, and direct the $z$ axis along the normal to this plane. Let the movement of the base under a kinematic perturbation be carried out with frequency and amplitude $A$, i.e.

$$
\omega_{k}(t)=A_{p} \sin p t
$$

Let us place a sufficient number of small elastically attached masses of absorbers above the plate so that their action on it can be considered distributed (Fig.1).

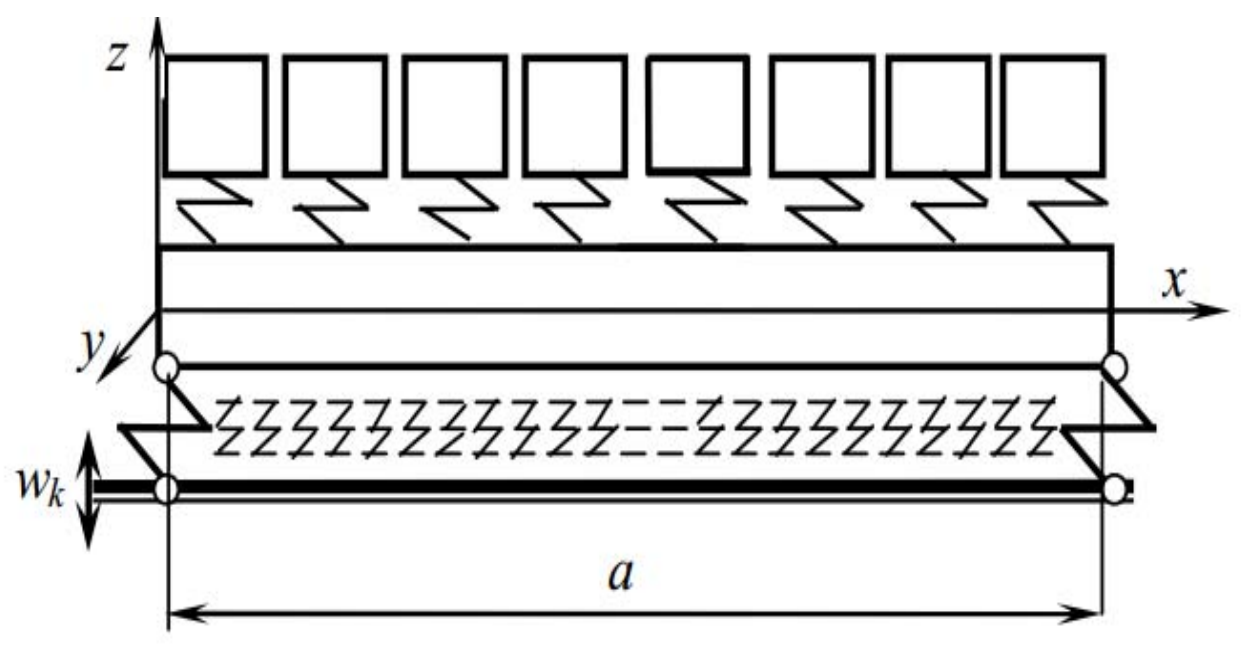

Fig.1. The number of small elastically attached masses of absorbers above the plate.

In the resulting oscillatory system, one can consider a conventional layer of dynamic absorbers, each element of which performs independent movements orthogonally to the middle surface of the plate. The curved surface of a section that is sufficiently distant from the transverse edges of the long plate $(a>4.0 b)$ can be considered cylindrical [6]. Then mentally cutting out an elementary strip from the plate in the direction of the $x$ axis, the latter can be considered as a beam of length $l$ on a viscoelastic base.

The integro-differential equations of small vibrations of the plate near the position of static equilibrium 
for deflections $w$ and displacements of the absorber layer have the form $W_{0}[18]$

$$
\begin{aligned}
& m \frac{\partial^{2} \omega}{\partial t^{2}}+D \frac{\partial^{4} \omega}{\partial x^{4}}=-c_{\alpha 0}\left[\left(\omega-\omega_{p}\right)-\int_{-\infty}^{t} R_{\alpha 0}(t-\tau)\left(\omega-\omega_{p}\right) d \tau\right]+ \\
& -c_{g 0}\left[\left(\omega-\omega_{k}\right)-\int_{-\infty}^{t} R_{g 0}(t-\tau)\left(\omega-\omega_{k}\right) d \tau\right], \\
& \rho_{g} h_{g} \frac{\partial^{2} \omega_{k}}{\partial t^{2}}=-c_{g 0}\left[\left(\omega_{k}-\omega\right)-\int_{-\infty}^{t} R_{g 0}(t-\tau)\left(\omega_{k}-\omega\right) d \tau\right]
\end{aligned}
$$

where $\rho$ and $\rho_{g}$ - density of the plate material and layer of absorbers, respectively; $h$ and $h_{g}$ - thickness of the plate and layer of absorber, respectively; $c_{a 0}$ and $c_{g 0}$ - are the stiffness coefficients of the base of the plate and the layer of absorber, respectively.

The bending moment $M$ and the shearing force $Q$ in the plate section perpendicular to the $x$ axis are defined as $[3,19,20]$

$$
\begin{aligned}
& M=-\frac{E_{0} h_{0}^{3}}{12\left(1-v_{0}^{2}\right)}\left[\frac{\partial^{2} w}{\partial x^{2}}-\int_{-\infty}^{t} R_{D}(t-\tau) \frac{\partial^{2} w}{\partial x^{2}} d \tau\right] \\
& Q=-\frac{E_{0} h_{0}^{3}}{12\left(1-v_{0}^{2}\right)}\left[\frac{\partial^{3} w}{\partial x^{3}}-\int_{-\infty}^{t} R_{D}(t-\tau) \frac{\partial^{3} w}{\partial x^{3}} d \tau\right]
\end{aligned}
$$

where $E_{0}$ - instant modulus of elasticity of the plate, $h_{0}$ - plate thickness, $v_{0}$ - Poisson's ratio of the plate, $f(t)$ - time derivative. Then the boundary conditions at the longitudinal edges of the plate are:

$$
\begin{aligned}
& \left.\frac{\partial^{2} w}{\partial x^{2}}\right|_{x=0, a}=0 \\
& \left.\frac{\partial^{3} w}{\partial x^{3}}\right|_{x=0, a}= \pm \frac{12\left(1-v_{0}^{2}\right)}{E_{0} h_{0}^{2}} C_{0}\left[\left(w(x, t)-w_{p}(x, t)\right)+\right. \\
& \left.-\int_{-\infty}^{t} R_{C}(t-\tau)\left(w(x, \tau)-w_{p}(x, \tau)\right) d \tau\right]\left.\right|_{x=0, a}
\end{aligned}
$$

where $C_{0}$ - instant stiffness coefficient, $R_{c}(t-\tau)$ - relaxation core.

Thus, we have obtained a system of integro-differential equations (2.2) and the corresponding boundary conditions (2.4). 


\section{Solution methods}

Separating the variables according to the Fourier method for the steady-state vibration mode, we represent the plate deflections and the displacements of the absorber layer in the form of products of two functions

$$
w(x, t)=W(x) e^{-i w t}, \quad w_{0}(x, t)=W_{0}(x) e^{-i w t}
$$

where $W(x)$ and $W_{0}(x)$ - complex values of amplitude deflections and displacements, respectively, $w$ - vibration frequency.

After substituting them into the differential equations of oscillations we get:

$$
\begin{aligned}
& D W^{i v}+\left(c_{\alpha 0} \Gamma_{\alpha 0}+C_{g 0} \Gamma_{\alpha 0}-m \omega^{2}\right) W=c_{g 0} \Gamma_{g 0} W_{0}+c_{\alpha 0} \Gamma_{\alpha 0} R, \\
& \left(c_{g 0} \Gamma_{g 0}-\left(m \omega^{2}\right)\right) W_{0}=c_{g 0} \Gamma_{g 0} W, \\
& D=\frac{E_{0} h_{0}^{3}}{12\left(1-v_{0}^{2}\right)}, \quad \Gamma_{\alpha 0}=1-\Gamma_{\alpha 0}^{c}(\omega)-i \Gamma_{0}^{S}\left(\omega_{0}\right), \\
& \Gamma_{g 0}=1-\Gamma_{\alpha 0}^{c}(\omega)-i \Gamma_{0}^{s}\left(\omega_{0}\right)
\end{aligned}
$$

where

$$
\begin{array}{ll}
\Gamma_{\alpha 0}^{c}(\omega)=\int_{0}^{\infty} R_{\alpha 0}(\tau) \cos \omega \tau d \tau, & \Gamma_{\alpha 0}^{s}(\omega)=\int_{0}^{\infty} R_{\alpha 0}(\tau) \sin \omega \tau d \tau, \\
\Gamma_{g 0}^{c}(\omega)=\int_{0}^{\infty} R_{g 0}(\tau) \cos \omega \tau d \tau, & \Gamma_{g 0}^{s}(\omega)=\int_{0}^{\infty} R_{g 0}(\tau) \sin \omega \tau d \tau
\end{array}
$$

where Eqs (3.4) are cosine and sine of Fourier transform, respectively and $\bar{\omega}_{R}-$ is the actual value. The final resolving equation of plate vibrations can be represented as

$$
W^{i v}-\chi^{4} W=R c_{\alpha 0} \Gamma_{\alpha 0} D^{-1} \Gamma_{e 0}
$$

where

$$
\chi^{4}=m D^{-1} \omega^{2}\left(1+\frac{m_{0}}{m} \cdot \frac{\omega_{0}^{2}}{\omega_{0 \Gamma_{g 0}-\omega^{2}}^{2}} \Gamma_{g 0}\right)-c_{\alpha 0} \Gamma_{\alpha 0} D^{-1} \Gamma_{E 0}, \quad \omega_{0}=\sqrt{\frac{c_{g 0}}{m_{0}}},
$$

here $\omega_{0}$ - quencher layer partial frequency. 
It is convenient to represent the solution of Eq.(3.5) in the Krylov functions [1], which form a system of particular solutions with the unit matrix of the initial boundary conditions, which are taken in the form

$$
\begin{array}{ll}
U_{1}(x)=0.5(\operatorname{ch} \chi x+\cos \chi x), & U_{2}(x)=0.5(\operatorname{sh} \chi x+\sin \chi x), \\
U_{3}(x)=0.5(\operatorname{ch} \chi x-\cos \chi x), & U_{4}(x)=0.5(\operatorname{sh} \chi x-\sin \chi x),
\end{array}
$$

moreover

$$
U_{1}^{\prime}=\chi U_{4}, \quad U_{2}^{\prime}=\chi U_{1}, \quad U_{3}^{\prime}=\chi U_{2}, \quad U_{4}^{\prime}=\chi U_{3} .
$$

Introducing constants

$$
\begin{aligned}
& A_{1}=W(0), \quad A_{2}=W^{\prime}(0) \chi^{-1}, \quad A_{3}=W^{\prime \prime}(0) \chi^{-2}, \quad A_{4}=W^{\prime \prime \prime}(0) \chi^{-3}, \\
& p=c D^{-1} \chi^{-4}, \quad P=C D^{-1} \chi^{-3},
\end{aligned}
$$

the general solution of Eq.(3.4) can be represented as

$$
W(x)=A_{1} U_{1}(x)+A_{2} U_{2}(x)+A_{3} U_{3}(x)+A_{4} U_{4}(x)+p R\left[U_{1}(x)-1\right] .
$$

Considering the initial boundary conditions

$$
\begin{aligned}
& W^{\prime \prime}(0)=0, \quad W^{\prime \prime \prime}(0)=\bar{C}_{p} D^{-1}[W(0)-R], \quad \bar{C}_{p}=C_{0} \Gamma_{c}, \\
& \Gamma_{C}=1-\Gamma_{c 0}^{C}(\omega)-i \Gamma_{c 0}^{S}(\omega), \quad A_{3}=0, A_{4}=\left(A_{1}-R\right) P .
\end{aligned}
$$

Here $\Gamma_{c 0}^{C}(\omega), \Gamma_{c 0}^{S}(\omega)$ are defined as Eq.(3.3).

In this case, the shape of the plate deflections is

$$
W(x)=A_{1}\left[U_{1}(x)+P U_{4}(x)\right]+A_{2} U_{2}(x)+\left[p U_{1}(x)-p-P U_{4}(x)\right] R .
$$

Considering the final boundary conditions

$$
W^{\prime \prime}(a)=0, \quad W^{\prime \prime \prime}(a)=-\bar{C}_{p} D^{-1}[W(a)-R]
$$

we get the equations

$$
\begin{aligned}
& A_{1}\left[U_{3}(a)+P U_{2}(a)\right]+A_{2} U_{4}(a)=\left[P U_{2}(a)-p U_{3}(a)\right] R, \\
& \left.A_{1}\left[U_{2}(a)+2 P U_{1}(a)\right]+P^{2} U_{4}(a)\right]+A_{2}\left[U_{3}(a)-P U_{2}(a)\right]= \\
& =\left\{(1+p) P+(1-p) P U_{1}(a)-p U_{2}(a)+P^{2} U_{4}(a)\right\} R,
\end{aligned}
$$


after which

$$
\begin{aligned}
& A_{1}=\frac{0.5 R}{A}[(1-p) P(\operatorname{ch} \chi \alpha \sin \chi \alpha-\operatorname{sh} \chi \alpha \cos \chi a)-(1+p) P(\operatorname{sh} \chi \alpha-\sin \chi \alpha)+ \\
& \left.-p(1-\operatorname{ch} \chi \alpha \cos \chi \alpha)+2 P^{2} \operatorname{sh} \chi \alpha \sin \chi \alpha\right]
\end{aligned}
$$

where

$$
A=0.5\left[(1-\operatorname{ch} \chi \alpha \cos \chi \alpha)+2 P(\operatorname{ch} \chi \alpha \sin \chi \alpha-\operatorname{sh} \chi \alpha \cos \chi \alpha)+2 P^{2} \operatorname{sh} \chi \alpha \sin \chi \alpha\right]
$$

Introducing dimensionless parameters

$$
\begin{aligned}
& \bar{\chi}=\chi \alpha, \quad \bar{C}=C_{0} D^{-1} a, \quad \bar{c}=c D^{-1} a^{4}, \quad \bar{c}_{0}=c_{0} D^{-1} a^{4}, \\
& \bar{\omega}^{2}=\omega^{2} p h D^{-1} a^{4}, \quad \bar{m}=m_{0}(p h)^{-1},
\end{aligned}
$$

then for the dimensionless stiffness $\bar{c}_{0}$ of absorbers, taking into account expression (3.4), we have

$$
\bar{c}_{0}=\bar{m} \bar{\omega}^{2}\left\{1-\bar{m}_{0}\left[\left(\bar{\chi}^{4}+\bar{c}\right) \bar{\omega}^{2}-1\right]^{-1}\right\}^{-1} .
$$

Complete damping of forced vibrations of the longitudinal edges of the plate will be provided $A_{1}=0$, which leads to the equation

$$
A_{c}\left(\chi, \bar{C}_{p}\right) \bar{\chi}^{4}-D_{c}\left(\chi, \bar{C}_{p}\right) \bar{\chi}^{3}+2 \bar{C}_{p}^{2} \bar{\chi}+B_{c}\left(\chi, \bar{C}_{p}\right)=0
$$

where

$$
\begin{aligned}
& A_{c}\left(\chi, C_{0}\right)=\left[\frac{1+\operatorname{ch} \bar{\chi}}{\operatorname{sh} \bar{\chi}}-\frac{1+\cos \bar{\chi}}{\sin \bar{\chi}}\right] C_{0} \Gamma_{c}^{\square}, \quad D_{c}(\chi, \bar{C})=\frac{1-\operatorname{ch} \bar{\chi} \cos \bar{\chi}}{\operatorname{sh} \bar{\chi} \sin \bar{\chi}} c_{\alpha 0} \Gamma_{c}^{\square}, \\
& B_{c}(\chi, \bar{C})=\left[\frac{1-\operatorname{ch} \bar{\chi}}{\operatorname{sh} \bar{\chi}}-\frac{1-\cos \bar{\chi}}{\sin \bar{\chi}}\right] c_{\alpha 0} C_{0} \Gamma_{C}^{\square} \Gamma_{c}^{\square}, \quad \bar{C}_{p}=C_{0} \Gamma_{c}^{\square}, \\
& \Gamma_{C}^{\square}=1-\Gamma_{C}^{c}(\chi)-i \Gamma_{c}^{s}(\chi), \\
& \Gamma_{c}^{\square}=1-\Gamma_{c}^{c}(\chi)-i \Gamma_{c}^{s}(\chi)
\end{aligned}
$$

where $\Gamma_{C}^{c}(\chi), \Gamma_{c}^{s}(\chi), \Gamma_{c}^{c}(\chi), \Gamma_{c}^{s}(\chi)$ are defined similarly Eq.(3.2). For given dimensionless instantaneous stiffnesses $C_{0}$ of viscoelastic supports and viscoelastic foundation $c_{0}$, the complex parameter is determined from the solution of the equation by a complex output parameter $\bar{\chi}$. 


\section{Numerical results}

As the initial data, you should specify the radii (large and small), the height and thickness of the shell, the angle of the half of the truncated cone, the modulus of elasticity, Poisson's ratio, the parameters of the relaxation kernel of the material, and the geometric and mechanical parameters of the ribs. As the relaxation kernel of a viscoelastic material, we take the three-parameter kernel $R(t)=\left(A e^{-\beta t}\right) t^{-(1-\alpha)}$. RzhanitsynKoltunova $[21,22,23,24]$, which has a weak singularity. Here $A, \beta, \alpha$ are-material parameters. We take the parameter values in the form:

$$
\begin{aligned}
& A=0.048, \quad \beta=0.05, \quad \alpha=0.1, \quad C_{0}=50, \quad c_{\alpha 0}=25(\chi=2.959), \\
& a=2 \quad \text { and } \quad \bar{m}_{0}=0.025
\end{aligned}
$$

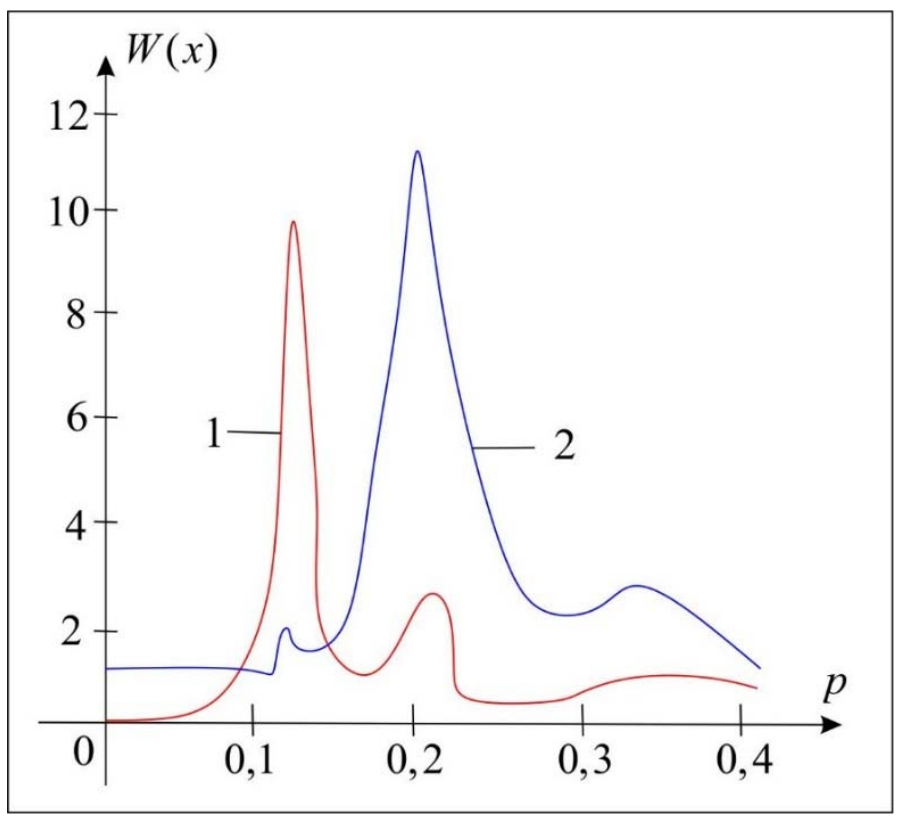

Fig.2. The amplitude and frequency characteristics of the point $\left(x=\frac{a}{2}\right)$ at the frequency of vibration loads.

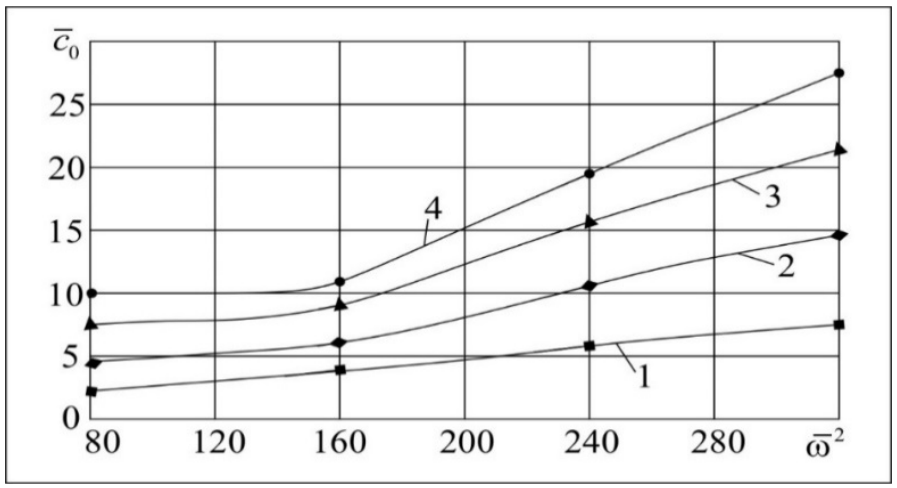

Fig.3. Results of the corresponding calculations. 
Figure 2 shows the amplitude and frequency characteristics of the point $\left(x=\frac{a}{2}\right)$ at the frequency of vibration loads ( 1 - taking into account the viscosity of the deformed element, 2 - without taking into account the viscosity).

The figure shows that taking into account the viscous property of the deformed element (spring) reduces the displacement amplitudes to $15 \%$. For the given dimensionless stiffness of elastic supports $\bar{C}$ and an elastic foundation $\bar{C}$, the parameter of the plate $\bar{\chi}$ can be found from the solution of Eq.(3.2) [25, 26]. Further, choosing the ratio of the masses $\bar{m}_{0}$ of the absorbers and the plate, the required dimensionless stiffness $\bar{c}_{0}$ of the absorbers is determined by formula (3.5), depending on the specific dimensionless frequency of vibration of the base $\bar{\omega}$.

The results of the corresponding calculations are presented in the graphs in Fig.3. Thus, the correctness of the calculation of deformations and stresses in technical structures arising from bending vibrations depends on the assessment of the effect of small masses of absorbers on the vibration modes of the plate. Dependencies $\bar{c}_{0}$ from $\bar{\omega}^{2}$ for $\bar{C}=100$ and $\bar{c}=50(\chi=2.959)$ at $\bar{m}_{0}=0.025(1), 0.050(2), 0.075(3), 0.100(4)$.

The graphs presented can be used to adjust the dynamic masses of the absorbers, at which the longitudinal edges of the plate remain stationary.

If they are present, it is possible to determine their corresponding stiffness from a given frequency of kinematic disturbance at a selected mass of absorbers.

\section{Conclusions}

The ratio of the masses of the absorbers and the plate, the required dimensionless stiffness of the absorbers, depending on the specific vibration frequency of the base, are determined. The amplitude - frequency characteristics of the midpoint of the plate are constructed with and without regard to the viscosity of the deformed element. To ensure optimal damping of vibrations of the plate, the dependence of the stiffness of the deformed element on the frequency of the external action is obtained.

1. It is necessary to ensure, at high viscosity, that the resonant frequencies are very close to unity (the first natural frequency of the mechanical system), the dimensionless frequency at which it is necessary to damp the displacement amplitudes of the oscillatory mechanical system.

2. The most effective (in terms of optimal damping) is the use of two dynamic dampers for vertical movement.

3. To achieve optimal damping, one of the dampers must be installed at the center of gravity. The second damper must be removed as far as possible from the first. It can be seen that taking into account the viscous properties of the deformed element (spring) reduces the displacement amplitudes to $15 \%$.

\section{Nomenclature}

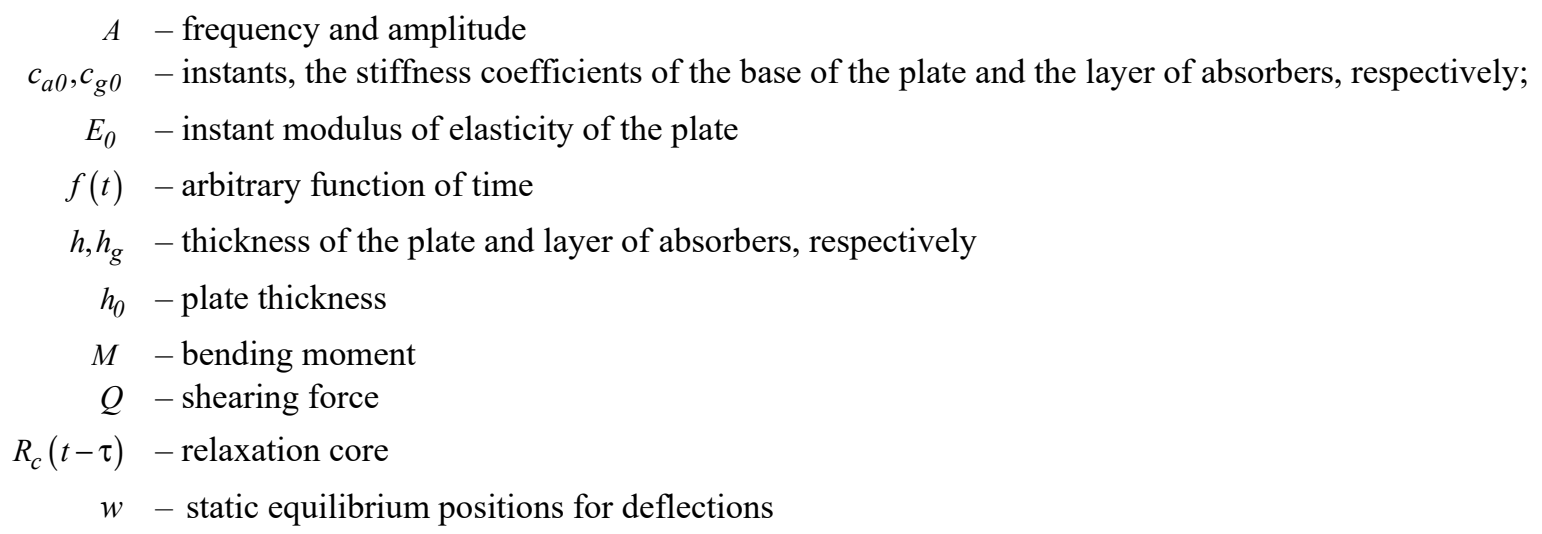




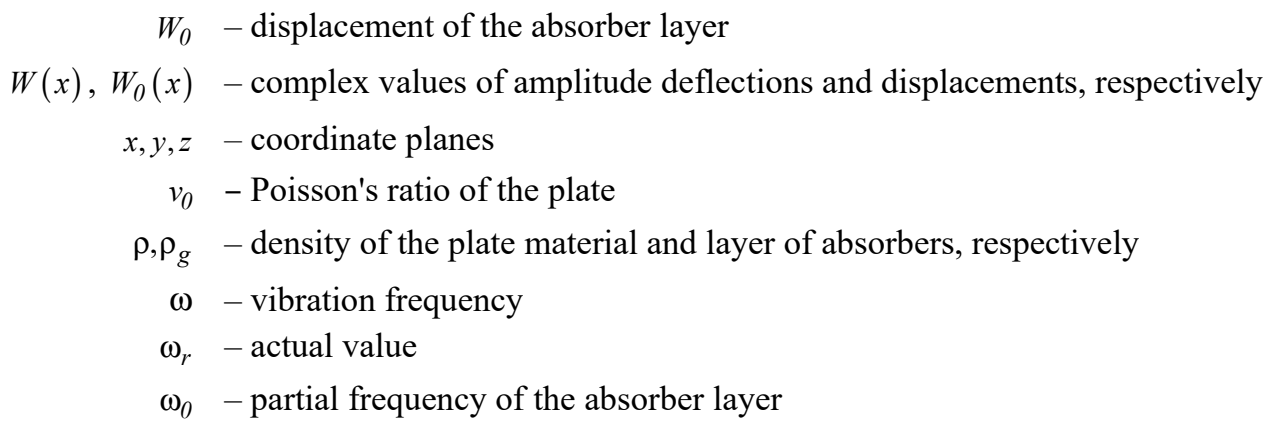

\section{References}

[1] Frolova KV (ed) (1981): Vibration in Technology: Handbook.- In: Protection from Vibration and Shock, Frolova KV (ed), Mechanical Engineering, Moscow, vol.6.

[2] Tokarev M.F., Talitskiy E.N. and Frolov V.A. (1984): Mechanical influences and protection of electronic equipment (in Russian).- Moscow, Radio and Communication.

[3] Nashif A.D., Jones D.I. and Henderson J.P. (1985): Vibration Damping.- John Wiley and Sons.

[4] Mirsaidov M.M., Safarov I.I. and Teshaev M.Kh. (2019): Oscillations of multilayer viscoelastic composite toroidal pipes.- Journal of the Serbian Society for Computational Mechanics, vol.13, No.2, pp.105-116 10.24874/jsscm.2019.13.02.08.

[5] Maiboroda V.P., Troyanovskii I.E., Safarov I.I., Vazagashvili M.G. and Katalymova I.V. (1992): Wave attenuation in an elastic medium.- Journal of Soviet Mathematics, vol.60, pp.1379-1382.

[6] Maiboroda V.P., Safarov I.I. and Troyanovskii I.E. (1983): Free and forced oscillations of a system of rigid bodies on inhomogeneous viscoelastic snubbers.- Soviet Machine Science, pp.25-31.

[7] Mirsaidov M.M., Safarov I.I., Teshaev M.K. and Boltayev Z.I. (2020): Dynamics of structural - inhomogeneous coaxialmulti-layered systems cylinder-shells.- Journal of Physics: Conference Series, vol.1706, No.1, Article number: 012033.

[8] Marano G.C., Greco R. and Sgobba S. (2010): A comparison between different robust optimum design approaches: Application to tuned mass dampers.- Probabilistic Engineering Mechanical, vol.25, pp.108-118. https://doi.org/10.1016/j.probengmech.2009.08.004

[9] Qiu Z. and Wang X. (2003): Comparison of dynamic response of structures with uncertain-but-bounded parameters using no probabilistic interval analysis method and probabilistic approach.- Int. J. Solids Struct., vol.40, pp.5423-5439. https://doi.org/10.1016/S0020-7683(03)00282-8.

[10] Muscolino G. and Sofi A. (2013): Bounds for the stationary stochastic response of truss structures with uncertain-butbounded parameters.- Mech. Syst. Signal Process, vol.37, pp.163-181, https://doi.org/10.1016/j.ymssp.2012.06.016.

[11] Antoniadis I.A., Kanarachos S.A., Gryllias K. and Sapountzakis I.E. (2018): KDamping: A stiffness based vibration absorption concept.- Journal Vib. Control, vol.24, pp.588-606, https://doi.org/10.1177/1077546316646514.

[12] Kofanov Yu.N., Shalumov A.S., Goldin V.V. and Zhuravsky V.G. (2000): Mathematical modeling of radio-electronic devices under mechanical influences.- Moscow: Radio and Communication, p.226.

[13] Capatti M.C., Carbonari S. and Gara F. (2016): Experimental study on instrumented micro piles.- Environmental, Energy and Structural Monitoring Systems (EESMS), 2016 IEEE Workshop, pp.1-6, DOI: 10.1109/EESMS.2016.7504831.

[14] Adamo F. (2014): Assessment of the uncertainty in human exposure to vibration: an experimental study.- IEEE Sensors Journal, vol.14, No.2, pp.474-481. DOI: 10.1109/JSEN.2013.2284257.

[15] Palacios-Quiñonero F. and Karimi H.R. (2013): Passive-damping design for vibration control of large structures.Control and Automation (ICCA), 10th IEEE International Conference, pp.33-38. DOI: 10.1109/ICCA.2013.6565018.

[16] Zhang X., Sun D., Song Y. and Yan B. (2010): Dynamics characteristic study of the visco-elastic suspension system of construction vehicles.- Technology and Innovation Conference, ITIC 2009, pp.1-4. DOI: 10.1049/cp.2009.1508.

[17] Sahu S.K. and Datta P.K. (2003). Dynamic stability of laminated composite curved panels with cutouts.- Journal of Engineering Mechanics, vol.29, No.11, pp.1245-1253.

[18] Korenev B.G. and Reznikov L.M. (1988): Dynamic Vibration Dampers: Theory and Technical Applications.Science, Fizmatgiz, Moscow. 
[19] Il'yushin A.A. and Pobedrya B.E. (1970): Fundamentals of the Mathematical Theory of Thermoviscoelasticity.- Science, Moscow, p.280.

[20] Mirsaidov M., Safarov I. and Teshaev M. (2020): Dynamic instability of vibrations of thin-wall composite curvorine viscoelastic tubes under the influence of pulse pressure.- E3S Web of Conferences, pp.164.

[21] Koltunov A.A. and Maksudov R. (1976): Dynamic stability of a flexible viscoelastic cylindrical shell.- Polymer Mechanics, vol.12, No.5, pp.829-831.

[22] Safarov I., Teshaev M., Toshmatov E., Boltaev Z. and Homidov F. (2020): Torsional vibrations of a cylindrical shell in a linear viscoelastic medium.- IOP Conference Series: Materials Science and Engineering, vol.883, No.1, Article number 012190.

[23] Sayfidinov O. and Bognár G.V. (2021): Numerical solutions of the Kardar-Parisi-Zhang interface growing equation with different noise terms.- In: Jármai K. and Voith K. (eds) Vehicle and Automotive Engineering 3, VAE 2020, Lecture Notes in Mechanical Engineering, Springer, Singapore. https://doi.org/10.1007/978-98115-9529-5_27.

[24] Mirsaidov M., Safarov I., Boltayev Z. and Teshaev M. (2020): Spread waves in a viscoelastic cylindrical body of a sector cross section with cutouts.- IOP Conference Series: Materials Science and Engineering, vol.869, No.4, Article number 042011.

[25] Boltaev Z., Safarov I. and Razokov T. (2020): Natural vibrations of spherical inhomogeneity in a viscoelastic medium.- International Journal of Scientific and Technology Research, vol.9, No.1, pp.3674-3680.

[26] Teshaev M., Safarov I.I. and Mirsaidov M. (2019): Oscillations of multilayer viscoelastic composite toroidal pipes.J. Serbian Soc. Comput. Mech., vol.13, pp.104-115.

Received: January 19, 2021

Revised: $\quad$ March 18, 2021 